\title{
Primary Lymphoma of the Calcaneus: A Case Report
}

\author{
Khodamorad Jamshidi, ${ }^{1}$ and Farshad Abolghasemzadeh Ahangar ${ }^{1,}{ }^{*}$ \\ ${ }^{1}$ Bone and Joint Reconstruction Research Center, Shafa Orthopedic Hospital, Iran University of Medical Sciences, Tehran, IR Iran \\ "Corresponding author: Farshad Abolghasemzadeh Ahangar, Bone and Joint Reconstruction Research Center, Shafa Orthopedic Hospital, Iran University of Medical Sciences, \\ Tehran, IR Iran. Tel: +98-9124454805, Fax: +98-2133542022, E-mail: farshada1358@gmail.com
}

Received 2016 April 02; Revised 2016 June 01; Accepted 2016 July 08.

\begin{abstract}
Introduction: Primary Non-Hodgkin's lymphoma (NHL) of the bone is a rare condition. Calcaneus is also an uncommon site for this kind of lymphoma.

Case Presentation: We hereby present a case of primary non-Hodgkin's lymphoma of the calcaneus, which was diagnosed and treated at our hospital. The patient had a minor twisting trauma of his ankle but symptoms were present for one year after trauma. The patient complained from instability and swelling of his ankle. After paraclinic assessments, a pathologic lesion was detected in his calcaneus, so incisional biopsy was done and the pathology diagnosis was a large B-cell non-Hodgkin's lymphoma. Bone marrow assessment was negative for the disease. The patient was treated by chemotherapy and radiotherapy and currently the disease is in remission and patient is doing his previous full activity without any important complication.

Conclusions: Lymphoma is a rare condition in the foot region but we suggest to be more wise for chronic, uncommon symptoms after minor injuries, as lymphoma of the bone can present mysterious and nonspecific symptoms as was seen in this patient. It may be better to do more assessments to prevent delayed diagnosis of such rare conditions. These rare cases may have atypical imaging and clinical appearance, which can lead to misdiagnosis.
\end{abstract}

Keywords: Lymphoma, Calcaneus, Diagnosis

\section{Introduction}

Primary Non-Hodgkin's lymphoma (NHL) of the bone is a rare condition. It accounts for less than $2 \%$ of all lymphomas in adults (1). In the pediatric population, it represents about 3\% to 9\% of NHL cases (2-5). It is slightly more frequent in men than women with male: female ratio ranging from 1.2 to 1.8 (6-9). Most of the patients are over the age of 30 years $(92 \%)(6-8)$.

The femur (29\%) is the most common site for primary lymphoma of the bone, followed by pelvis (19\%), humerus (13\%), skull (11\%) and tibia (10\%) (10).

As mentioned above, calcaneus is a very uncommon site for primary bone lymphoma. Rare cases of calcaneal lymphoma have been reported in the literature (11). Here we present a case of primary non-Hodgkin's lymphoma of the calcaneus, which was diagnosed and treated at our hospital.

\section{Case Presentation}

This study was approved by the ethics board of our institution and parents of our patients gave their written consent to publish the case report. The patient was a 13-year-old boy, who complained from chronic pain and swelling around his right ankle. There was a history of ankle twisting injury before the onset of symptoms approximately one year before admission to our hospital. After the mentioned trauma, a three-week period of short leg cast was done for ankle sprain as the diagnosis. However, after cast removal the patient had multiple (five times) twisting incidences at the same ankle and after each twisting he experienced a few weeks of pain and swelling, which were self-limited. Between twists, the patient had no sign or symptoms and had his full daily activities.

Ultimately, the patient was referred to our hospital with chronic pain and instability of ankle as chief complaints. We assessed the patient and imaging changes like a tumoral involvement in his calcaneus was detected (Figure 1). Thus, we carried out a complete tumor work-up consisting of magnetic resonance imaging (MRI), whole body bone scan, chest computerized tomography (CT)-scan and laboratory tests (complete blood count (CBC) and differentiation, alkaline phosphatase, Calcium, Phosphorous, Erythrocyte Sedimentation Rate (ESR) and C-Reactive protein (CRP)). The lesion had markedly increased uptake of Technetium-99m in bone scintigraphy (Figure 2). The extension of the involved area was better seen in the MRI (Figure 3). The lesion involved the subchondral area of the subtalar joint and anterior portion of the calcaneus. All labo- 
Figure 1. Lateral X-Ray of the Calcaneus One Year After the First Twisting When the Patient was Admitted to Our Hospital

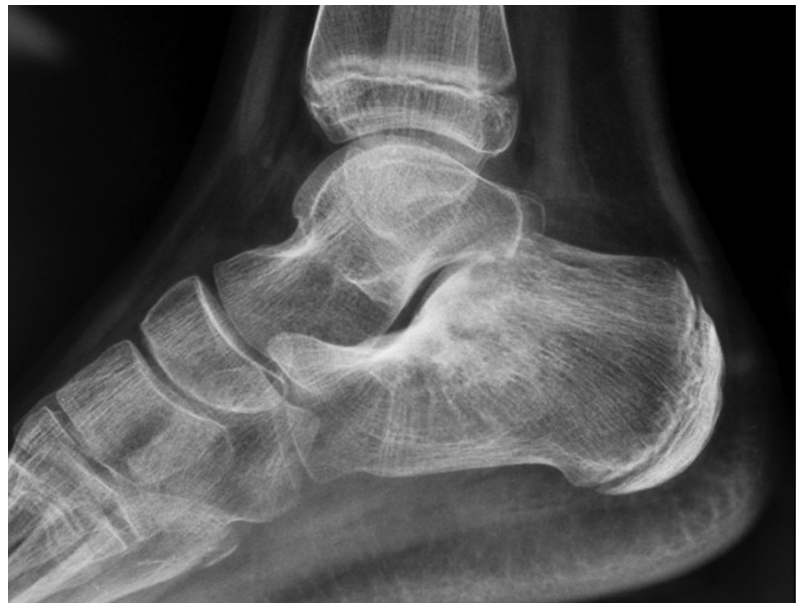

Sclerotic changes can be seen in subchondral area of the subtalar joint in the calcaneus.

ratory tests and chest CT-scan were normal.

After complete assessment, the patient was scheduled for incisional biopsy. A loose myxoid brown tissue was achieved. According to pathology report, diagnosis was a large B-cell non-Hodgkin's lymphoma that was approved by the IHC (Figure 4). Furthermore, systemic assessment of the patient was done by bone marrow aspiration and CTscan from abdomen and pelvis. There was no systemic involvement in bone marrow or lymph nodes. Thus it was diagnosed as a primary lymphoma of the bone.

We decided to treat this patient by chemotherapy and radiotherapy. We referred the patient to an oncologist and a radiotherapist. The patient underwent courses of five drugs (RCHOP) chemotherapy followed by 25 sessions of

Figure 2. Bone Scan of the Affected Area

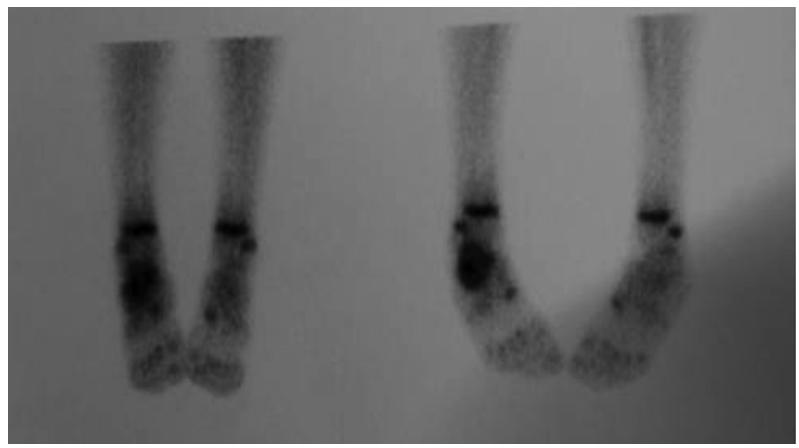

Note the increased uptake in the right calcaneus.
Figure 3. Sagittal Cut of the Magnetic Resonance Imaging (T1 Weighted)

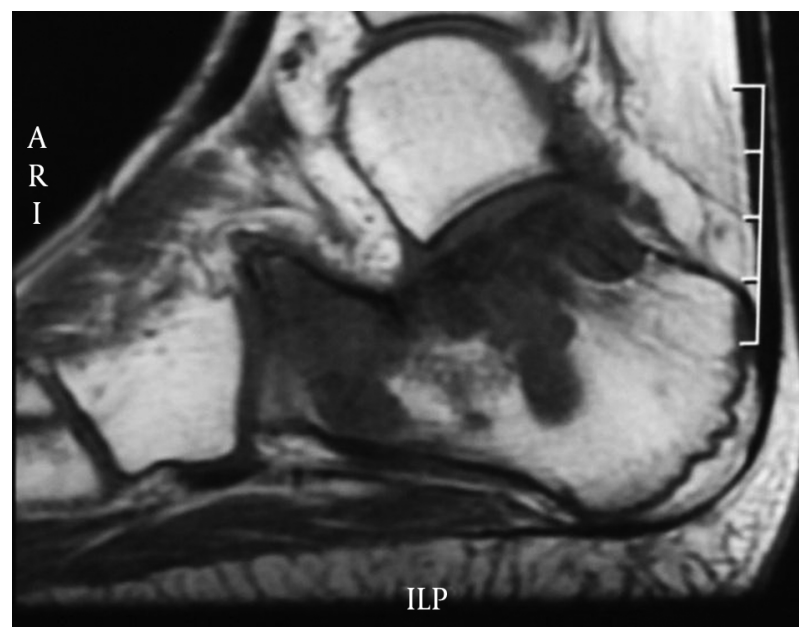

Note the dark signal of the affected area

radiotherapy. Then patient was under close follow-up every three months. After 14 months of follow-up, the disease was in remission and patient had no signs or symptoms of recurrence. He has a painless full weight bearing gait and has returned to his previous activities. The only complaint is slight painless swelling around the ankle after long standing or running.

Figure 4. Pathologic Photography of the Cytologic Assessment (Intermediate Power $* 40$, H and E Staining)

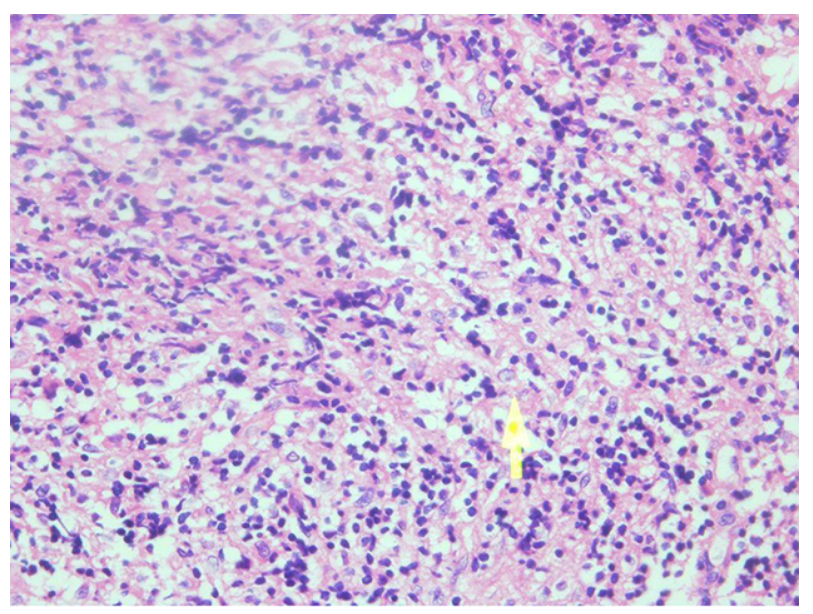

A large number of small blue cells are seen in a loose eosinophilic stroma (arrow). 


\section{Discussion}

Primary lymphoma of the bone is a rare entity and more uncommon in calcaneus (8-10). This can be a cause of delayed diagnosis or misdiagnosis in these cases. Although the imaging appearance of primary bone lymphoma is variable and nonspecific, its typical picture is a solitary lytic lesion near the end of long bones, yet this typical appearance may not be visible at other uncommon sites. Most common sites for presentation of lymphoma of the bone are femur, pelvis, humerus, skull and tibia (10). However, any part of the calcaneus can be involved by lymphoma (8-10). Distribution of the tumor along the soft tissue attachments like Achilles tendon was reported (9).

Generally, primary signs and symptoms of the lymphoma in calcaneus are not specific. Mild nonspecific pain after daily activity is often the chief complaint. Mild swelling and non-pitting edema around the heel were two prevalent symptoms (9). In about half of cases, a history of trauma was reported. Almost all patients had good general condition at the time of diagnosis (6-10). As in our patient, in another three cases, relative instability in the ankle was reported $(9,10)$.

In our case, a history of ankle twisting confused previous physicians whom had visited the patient during the year from the onset of symptoms to the diagnosis. Also, the age of the patient was not in the common age range for this diagnosis. After tumor work-up, our differential diagnosis for the lesion was infection and Ewing sarcoma. Of course, normal CBC, ESR and CRP were against both diagnoses. In contrast to the expected typical imaging appearance for primary bone lymphoma, in this case an illdefined sclerosis was seen in the subchondral area of the subtalar joint, where it diffused into the body of the calcaneus (Figure 1). Reviewing previous radiographies, the first radiography at the first twisting (Figure 5) of the ankle seemed normal and without any visible changes. However, the second radiography (Figure 6), which was taken for the 4th twisting, six months later, showed sclerotic changes but unfortunately was not attended and finally after the 5th twisting when MRI was requested, the lesion was detected. The MRI showed obvious signal changes and the extension of the disease was defined. Common treatment for isolated primary bone lymphomas is chemotherapy alone or in combination with radiotherapy (2-4). However, there are rare reports of surgical excision before chemotherapy (11). We treated this patient through the protocol of our center for primary bone lymphomas, which is a combination of chemotherapy and radiotherapy. Until the time of this case report, the disease was at the remission state and the patient had no important morbidity.

According to this case report, it may be better to do
Figure 5. Radiography After the First Twisting

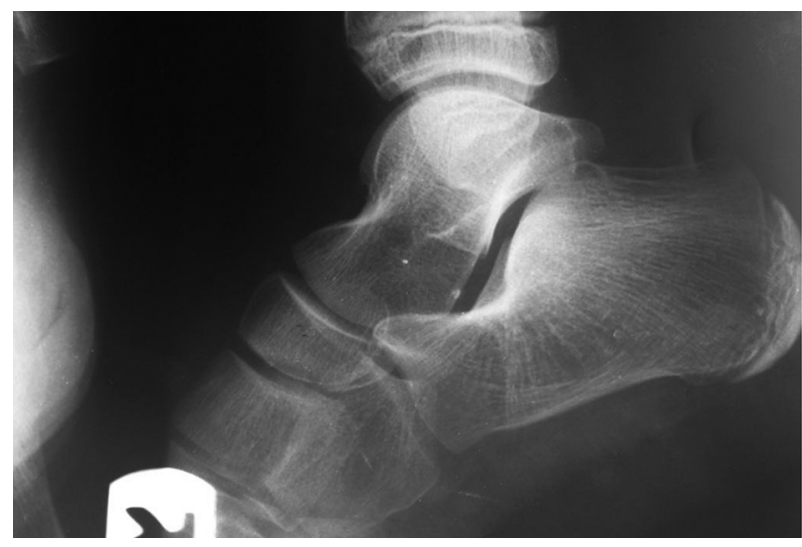

It can mimic a normal radiography. Sclerotic change in the subtalar area is not obvious.

Figure 6. Radiography After the Fourth Twisting (Six Months Before Admission)

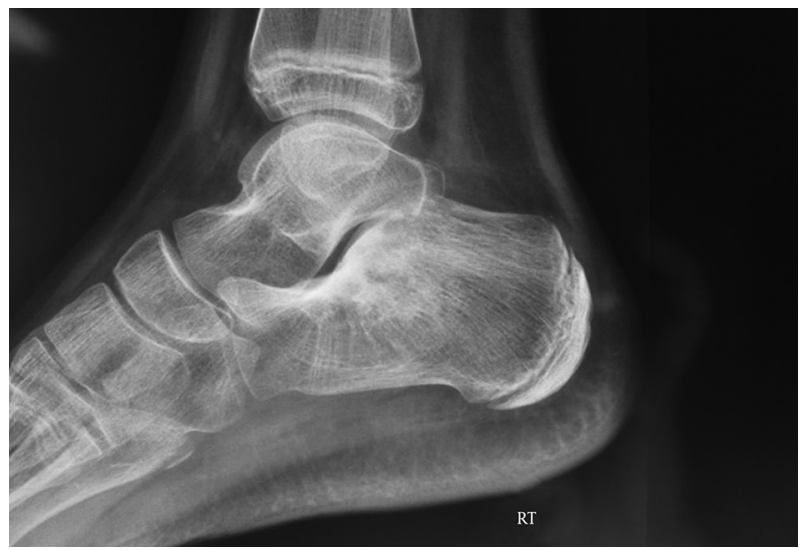

Now sclerotic changes caused by the tumor are obvious.

more assessment in patients, which complain of chronic symptoms after a minor injury and such rare conditions should always be in mind.

\section{Footnote}

Authors' Contribution: Khodamorad Jamshidi, diagnosis, treatment, concept and supervision; Farshad Abolghasemzadeh Ahangar, draft and revision of the manuscript.

\section{References}

1. Dubey P, Ha CS, Besa PC, Fuller L, Cabanillas F, Murray J, et al. Localized primary malignant lymphoma of bone. Int J Radiat Oncol Biol Phys. 1997;37(5):1087-93. [PubMed: 9169817]. 
2. Loeffler JS, Tarbell NJ, Kozakewich H, Cassady JR, Weinstein HJ. Primary lymphoma of bone in children: analysis of treatment results with adriamycin, prednisone, Oncovin (APO), and local radiation therapy. J Clin Oncol. 1986;4(4):496-501. [PubMed: 3958763].

3. Furman WL, Fitch S, Hustu HO, Callihan T, Murphy SB. Primary lymphoma of bone in children. J Clin Oncol. 1989;7(9):1275-80. [PubMed: 2769328].

4. Anderson JR, Wilson JF, Jenkin DT, Meadows AT, Kersey J, Chilcote $\mathrm{RR}$, et al. Childhood non-Hodgkin's lymphoma. The results of a randomized therapeutic trial comparing a 4-drug regimen (COMP) with a 10-drug regimen (LSA2-L2). N Engl J Med. 1983;308(10):559-65. doi: 10.1056/NEJM198303103081003. [PubMed: 6338381].

5. Lones MA, Perkins SL, Sposto R, Tedeschi N, Kadin ME, Kjeldsberg CR, et al. Non-Hodgkin's lymphoma arising in bone in children and adolescents is associated with an excellent outcome: a Children's Cancer Group report. J Clin Oncol. 2002;20(9):2293-301. [PubMed: 11981000].

6. Jawad MU, Schneiderbauer MM, Min ES, Cheung MC, Koniaris LG, Scully SP. Primary lymphoma of bone in adult patients. Cancer. 2010;116(4):871-9. doi: 10.1002/cncr.24828. [PubMed: 20043324].

7. Mulligan ME, McRae GA, Murphey MD. Imaging features of primary lymphoma of bone. AJR Am J Roentgenol. 1999;173(6):1691-7. doi: 10.2214/ajr.173.6.10584821. [PubMed:10584821].

8. Cai L, Stauder MC, Zhang YJ, Poortmans P, Li YX, Constantinou N, et al. Early-stage primary bone lymphoma: a retrospective, multicenter Rare Cancer Network (RCN) Study. Int J Radiat Oncol Biol Phys. 2012;83(1):284-91. doi: 10.1016/j.ijrobp.2011.06.1976. [PubMed: 22079728].

9. Bruno Ventre M, Ferreri AJ, Gospodarowicz M, Govi S, Messina C, Porter $\mathrm{D}$, et al. Clinical features, management, and prognosis of an international series of 161 patients with limited-stage diffuse large B-cell lymphoma of the bone (the IELSG-14 study). Oncologist. 2014;19(3):291-8. doi: 10.1634/theoncologist.2013-0249. [PubMed: 24567283].

10. Salter M, Sollaccio RJ, Bernreuter WK, Weppelmann B. Primary lymphoma of bone: the use of MRI in pretreatment evaluation. Am J Clin Oncol. 1989;12(2):101-5. [PubMed: 2705397].

11. Blume P, Charlot-Hicks F, Mohammed S. Case report and review of primary bone diffuse large B-cell lymphoma involving the calcaneus. J Foot Ankle Surg. 2013;52(5):666-72. doi: 10.1053/j.jfas.2013.03.013. [PubMed: 23628193]. 